Research Article

www.jestr.org

\title{
A Fast Neutron and Gamma Ray System for the Detection of Illicit Materials Based on Simple Isotopic Sources
}

\author{
J. G. Fantidis ${ }^{1, *}$, A. Dalakas ${ }^{1}$, C. Potolias ${ }^{1,2}$, K. Karakoulidis ${ }^{1,2}$ and P. Kogias ${ }^{1,2}$ \\ ${ }^{1}$ MSc in Innovation in Technology \& Entrepreneurship, Technological Education Institute of Eastern Macedonia and Thrace, Saint \\ Loucas, Kavala, Greece \\ ${ }^{2}$ Eastern Macedonia and Thrace Institute of Technology, St. Lucas, Kavala 65404, Greece \\ Received 14 February 2016; Accepted 21 December 2016
}

\begin{abstract}
A simple Fast Neutron Gamma Ray system based on a common neutron and gamma sources, namely $252 \mathrm{Cf}$ and $137 \mathrm{Cs}$, has been simulated using the MCNP4B Monte Carlo code. More than 160 materials from different categories were considered and the simulations showed that the examined facility offers remarkable benefit in discrimination between illicit and not illicit materials. The use of three beams, rather than two, further improves the material discriminator.
\end{abstract}

Keywords: FNGR Systems; MCNP4B; ${ }^{252} \mathrm{Cf},{ }^{137} \mathrm{Cs}$, illicit materials

\section{Introduction}

Abandoned landmines and terrorist bomb attacks are severe issues threatening our society. To counter this threat, several technologies have been developed to detect explosive detection systems. The detection of hidden explosives is a major analytical problem that requires highly sensitive and specific methods for its solution. The existing technologies for explosive detection can be divided into three categories: non-explosive component detection, vapor and trace detection, and bulk detection. Unfortunately there isn't method without both benefits and drawbacks. For this reasons the improvement of security systems -not only in the aviation industry, is compulsory. In this respect, the scientific focus on the development of new illicit materials detection technologies is significant [1-2].

In the present day many inspection systems are based on the dual energy method with a view to scan hand baggage, parcels and containers. The discrimination of materials is achieved when the inspected object is alternately irradiated by X-ray with different energies. By comparing the difference in attenuation coefficients between organic and inorganic materials for high and low energy X-ray, the dualenergy method has been widely applied in luggage X-ray inspection systems for the purpose of material discrimination. With this method is easy to discriminate not only metals from organic materials, but also high-atomic number metals such as lead and uranium from common metals such as iron and aluminium. However are almost unfeasible to differentiate a wide range of organic materials [1-8].

The limitations of X-ray systems have motivated the development of alternative methods, including those based on neutrons. Fast neutrons are suitable to explore bulky objects because of their high penetration. An excellent scientific review by Buffler [3] classifies neutron-based methods in seven general categories. Useful information about the neutron and photon based techniques for chemical explosives detection in air cargo there are in the recently review by Runkle et al. [9]. Neutrons based systems and techniques are superior to X-ray systems but have not implemented by the security industry. We agree entirely with Buffer and Tickner [10] that the reasons for this are the high cost and the complexity of the neutron based systems.

The purpose of this work is to evaluate if a simple Fast neutron Gamma Ray (FNGR) system based on a small isotopic sources can offer significant information for the detection of illicit materials. Previous simulations by Fantidis and Nicolaou [11] have shown the benefit of using three instead of two nuclear beams in the same system. Also many dual, triple and quadruple systems have been evaluated at the same article. In this work a ${ }^{252} \mathrm{Cf}$ source and a ${ }^{137} \mathrm{Cs}$ gamma ray source, have been simulated using the MCNP4B Monte Carlo [12] code with intention to assess a new FNGR system.

\section{Fast Neutron and Gamma Ray method}

For the ideal case of narrow-beam geometry in which scattered radiation do not reach the detector, the transmission of the monoenergetic fast neutrons and gamma or X-rays through the object of density $\rho$ and thickness $\mathrm{x}$ can be calculated by means of the following:

$$
\frac{I_{n}}{I_{n}^{0}}=e^{-\mu_{n} \rho x}
$$

and

- E-mail address: fantidis@yahoo.gr

ISSN: 1791-2377 @ 2016 Eastern Macedonia and Thrace Institute of Technology. All rights reserved. 


$$
\frac{I_{g}}{I_{g}^{0}}=e^{-\mu_{g} \rho x}
$$

for neutrons and gamma rays respectively. $\mu_{n}, \mu_{g}$ are the neutron and gamma ray mass-attenuation coefficients correspondingly, $I_{n}$ and $I_{g}$ are the measured yield through at the investigated object and $I_{n}^{0}, I_{g}^{0}$ are the measured yield without the object. The logarithmic ratio $\mathrm{R}$, of the neutron and gamma-ray attenuation coefficients is:

$$
R=\frac{\mu_{n}}{\mu_{g}}=\frac{\ln \frac{I_{n}}{I_{n}^{0}}}{\ln \frac{I_{g}}{I_{g}^{0}}}
$$

The ratio $R$ describes the material of the unknown object and is independent of its thickness.

The precise calculation of $\mathrm{R}$ values is necessary with the aim to distinguish the unknown object. The absence of monoenergetics sources leads to beam-hardening effects. In accordance with Liu et al. [7] there is an uncertainty of $1 \%$ on $I_{n} / I_{n}^{0}$ value and $0.1 \%$ on $I_{g} / I_{g}^{0}$ value. Using these values is easy to find the minimum and the maximum $R$ value for each material.

\section{Facility and sources}

The geometrical configuration of the facility used in the present work is schematically shown in Fig. 1 and is similar to the one described previously, in Ref. 11 and 13 by Fantidis and Nicolaou. A cylindrical steel collimator, with a length of $100 \mathrm{~cm}$ and diameter of $3 \mathrm{~cm}$, collimates a neutron or a $\gamma$-ray beam towards the object. The intensity of the beam transmitted through the object is calculated at the rectangular detector cell with dimensions $1.5 \times 1.5 \times 5 \mathrm{~cm}^{3}$. The cell is collimated by a steel cylindrical collimator having a length of $50 \mathrm{~cm}$ and a diameter of $1.5 \mathrm{~cm}$. The unknown object is considered in a cubic form with a side of $20 \mathrm{~cm}$ and it is symmetrically placed around the axis of the two collimators at a distance of $200 \mathrm{~cm}$ and $150 \mathrm{~cm}$ from the source and the detector cell respectively. An isotropic disc source, with diameter of $3 \mathrm{~cm}$, is considered in the simulations.

The ${ }^{252} \mathrm{Cf}$ neutron source is simulated like a Watt fission spectrum using the coefficients provided by the MCNP4B code. The source emits neutrons extends up to $10 \mathrm{MeV}$ with a mean energy at $2.3 \mathrm{MeV}$. Further to the neutron emission, ${ }^{252} \mathrm{Cf}$ emits photons with a mean energy of $0.8 \mathrm{MeV}$ [14]. ${ }^{137} \mathrm{Cs}\left(\mathrm{E}_{\gamma}=0.662 \mathrm{MeV}\right)$ was considered as gamma ray source. Just for the comparison two X-rays sources with 4 and $9 \mathrm{MeV}$ end point energies with Bremsstrahlung spectra were also simulated.

\section{Results and discussion}

Fig. 2 shows the thickness of 24 illicit and not illicit materials from different categories for $0.1 \%$ transmission for the simulated sources. Penetration capabilities were calculated using the F2 tally with NPS up to $6 \times 10^{7}$ histories yielding an accuracy $<1 \%$. In the case of neutrons there is good penetration in all cases and the penetration for the heavy materials (plutonium, uranium, gold, and lead) is excellent compared to gamma or X-ray sources. In the presence of organic materials neutrons have good penetration but gamma or X-rays sources gives excellent penetration capabilities.

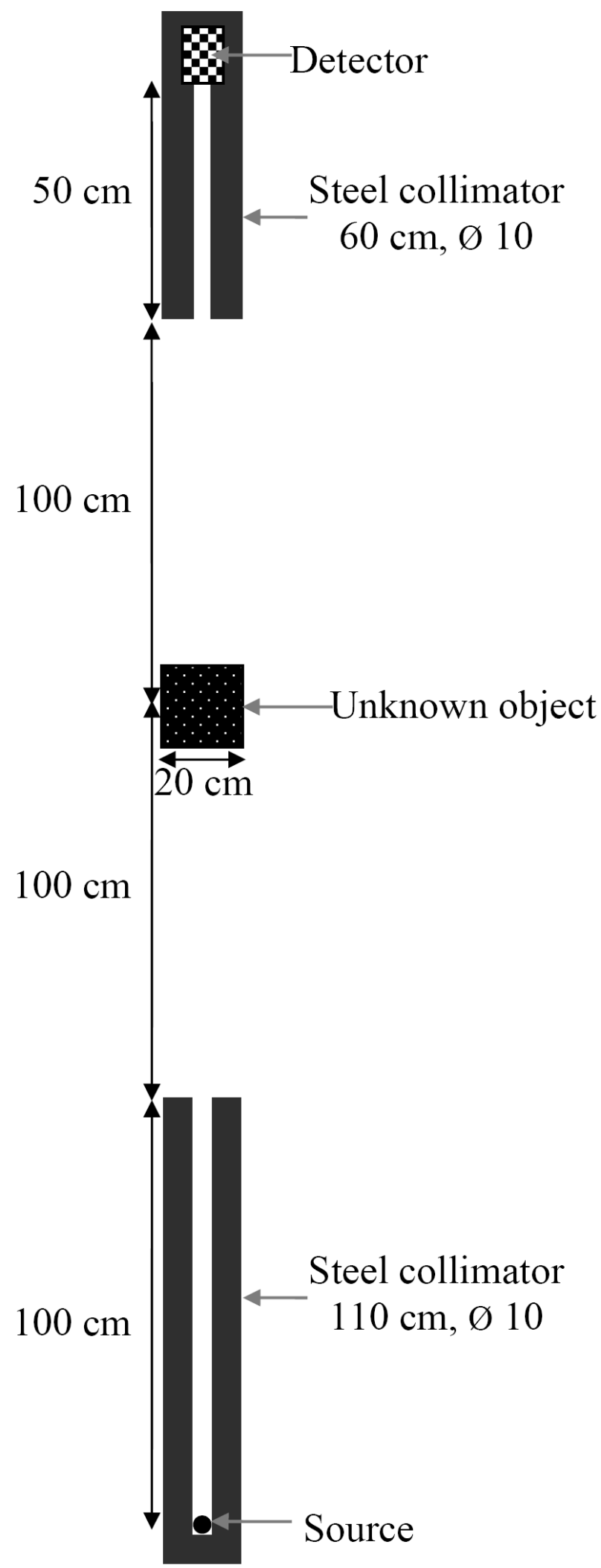

Fig. 1. The geometry for $R$ values calculations (not in scale). 


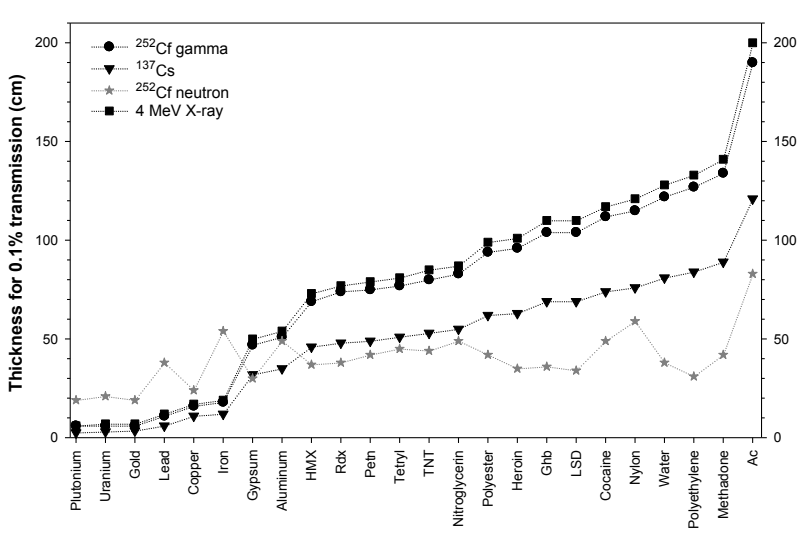

Material

Fig. 2. Thickness for 24 materials for $0.1 \%$ transmission for the 4 sources.

The R-values of the two simulated systems namely R1 $\left({ }^{252} \mathrm{Cf}\right.$ neutrons $/{ }^{252} \mathrm{Cf}$ gammas) and R2 $\left({ }^{252} \mathrm{Cf}\right.$ neutrons $\left./{ }^{137} \mathrm{Cs}\right)$, have been considered for an extensive series of illicit and not illicit materials, [15-52]. The simulated $\mathrm{R}$ values are given in Table 1. In Table 1, chemical weapons are presented in bold, explosives are highlighted in underlined letters, drugs are written in italics, and non- illicit materials are in normal letters.

The major advantage of the FNGR systems is the wide range of $R$ values (between polyethylene and the heaviest metals) which is also more uniformly stretch, with fine resolution between of organic materials and light and heavy metals. To demonstrate this, the two FNGR systems, compared with a dual beam X-ray system with 4 and $9 \mathrm{MeV}$ $\mathrm{X}$-ray sources. Fig. 3 illustrates the $R$ values for the 24 materials which studied before. It is clear that both FNGR systems achieve not only the good separation within the different classes of materials (organic materials, light and heavy materials) but also permit the discrimination between organic materials. The $R$ values were calculated with the MCNP4B code, using the *F1 tally card which gives the energy over a detector surface in $\mathrm{MeV}$. Calculations were carried out for NPS up to $8 \times 10^{9}$ histories yielding an accuracy of less than $0.08 \%$.

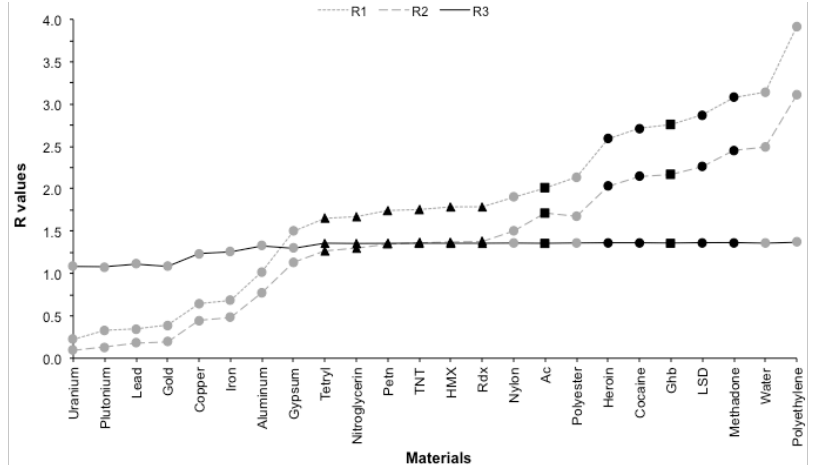

Fig. 3. The R values for the same 24 materials for different sources combinations.

Table 1. The R values of 165 materials for the 2 dual beam systems.

\begin{tabular}{|c|c|c|c|c|}
\hline Material & Density & Chemical Formula & $\mathbf{R}_{1}$ & $\mathbf{R}_{\mathbf{2}}$ \\
\hline Uranium & 18.97 & $\mathrm{U}$ & 0.222 & 0.093 \\
\hline Plutonium & 19.74 & $\mathrm{Pu}$ & 0.330 & 0.134 \\
\hline Bismuth & 9.75 & $\mathrm{Bi}$ & 0.345 & 0.175 \\
\hline Lead & 11.35 & $\mathrm{~Pb}$ & 0.347 & 0.178 \\
\hline Steel & 7.85 & From Ref. 53 & 0.354 & 0.249 \\
\hline Gold & 19.32 & $\mathrm{Au}$ & 0.392 & 0.193 \\
\hline Silver & 10.49 & $\mathrm{Ag}$ & 0.520 & 0.347 \\
\hline Copper & 8.92 & $\mathrm{Cu}$ & 0.645 & 0.448 \\
\hline Iron & 7.87 & $\mathrm{Fe}$ & 0.683 & 0.480 \\
\hline Silicon & 2.33 & $\mathrm{Si}$ & 0.911 & 0.699 \\
\hline Calcium & 1.54 & $\mathrm{Ca}$ & 0.967 & 0.621 \\
\hline Titanium Oxide & 4.17 & $\mathrm{TiO}_{2}$ & 0.972 & 0.719 \\
\hline Ck & 1.202 & $\mathrm{CNCl}$ & 0.978 & 0.782 \\
\hline $\mathrm{Cg}$ & 1.36 & $\mathrm{COCl}_{2}$ & 0.984 & 0.779 \\
\hline Dp & 1.656 & $\mathrm{C}_{2} \mathrm{Cl}_{4} \mathrm{O}_{2}$ & 0.991 & 0.776 \\
\hline Aluminum & 2.7 & $\mathrm{Al}$ & 1.023 & 0.776 \\
\hline Glass & 2.52 & $\mathrm{Si}_{4.21} \mathrm{Na}_{1.4} \mathrm{Al}_{0.1} \mathrm{O}_{9.96} \mathrm{Ca}_{0.63}$ & 1.032 & 0.777 \\
\hline $\mathbf{L}$ & 1.89 & $\mathrm{C}_{2} \mathrm{H}_{2} \mathrm{AsCl}_{3}$ & 1.062 & 0.837 \\
\hline Magnesia-Spinel Brick & 2.9 & $\mathrm{Mg}_{2.125} \mathrm{Al}_{0.294} \mathrm{O}_{2.567}$ & 1.065 & 0.803 \\
\hline Chloroform & 1.48 & $\mathrm{CHCl}_{3}$ & 1.099 & 0.871 \\
\hline Concrete & 2.2505 & From MCNP manual, Ref. 12 & 1.141 & 0.875 \\
\hline Hne & 1.85 & $\mathrm{C}_{2} \mathrm{~N}_{6} \mathrm{O}_{12}$ & 1.183 & 0.913 \\
\hline$\overline{\mathrm{Tnm}}$ & 1.64 & $\mathrm{CN}_{4} \mathrm{O}_{8}$ & 1.195 & 0.929 \\
\hline$\overline{\text { Md }}$ & 1.836 & $\mathrm{CH}_{3} \mathrm{AsCl}_{2}$ & 1.207 & 0.955 \\
\hline Dnaf & 1.91 & $\mathrm{C}_{4} \mathrm{~N}_{8} \mathrm{O}_{8}$ & 1.249 & 0.961 \\
\hline$\overline{\text { Pctfe }}$ & 2.13 & $\mathrm{C}_{2} \mathrm{ClF}_{3}$ & 1.268 & 0.977 \\
\hline Tntab & 1.74 & $\mathrm{C}_{6} \mathrm{~N}_{12} \mathrm{O}_{6}$ & 1.275 & 0.986 \\
\hline $\mathrm{Btf}$ & 1.86 & $\mathrm{C}_{6} \mathrm{~N}_{6} \mathrm{O}_{6}$ & 1.293 & 0.997 \\
\hline$\overline{\text { Teflon }}$ & 2.2 & $\mathrm{C}_{\mathrm{n}} \mathrm{F}_{2 \mathrm{n}+2}$ & 1.418 & 1.087 \\
\hline Ptfe & 2.15 & $\mathrm{C}_{2} \mathrm{~F}_{4}$ & 1.432 & 1.099 \\
\hline Dipso & 1.95 & $\mathrm{C}_{12} \mathrm{H}_{4} \mathrm{~N}_{6} \mathrm{O}_{14} \mathrm{~S}$ & 1.436 & 1.104 \\
\hline $\mathrm{Hl}$ & 1.6383 & $\mathrm{C}_{1.548} \mathrm{H}_{2.484} \mathrm{Cl}_{1.385} \mathrm{AS}_{0.306} \mathrm{~S}_{0.234}$ & 1.463 & 1.156 \\
\hline Nona & 1.7 & $\mathrm{C}_{18} \mathrm{H}_{5} \mathrm{~N}_{9} \mathrm{O}_{18}$ & 1.466 & 1.136 \\
\hline Ed & 1.742 & $\mathrm{C}_{2} \mathrm{H}_{5} \mathrm{AsCl}_{2}$ & 1.470 & 1.164 \\
\hline Dpo & 1.77 & $\mathrm{C}_{14} \mathrm{H}_{4} \mathrm{~N}_{8} \mathrm{O}_{13}$ & 1.475 & 1.141 \\
\hline$\overline{\text { Tnpy }}$ & 1.77 & $\mathrm{C}_{5} \mathrm{H}_{2} \mathrm{~N}_{4} \mathrm{O}_{6}$ & 1.478 & 1.142 \\
\hline Hnab & 1.6 & $\mathrm{C}_{12} \mathrm{H}_{4} \mathrm{~N}_{8} \mathrm{O}_{12}$ & 1.481 & 1.152 \\
\hline Pd & 1.645 & $\mathrm{C}_{6} \mathrm{H}_{5} \mathrm{AsCl}_{2}$ & 1.486 & 1.176 \\
\hline
\end{tabular}




\begin{tabular}{|c|c|c|c|c|}
\hline Material & Density & Chemical Formula & $\mathbf{R}_{1}$ & $\mathbf{R}_{2}$ \\
\hline Dips & 1.89 & $\mathrm{C}_{12} \mathrm{H}_{4} \mathrm{~N}_{6} \mathrm{O}_{12}$ & 1.497 & 1.152 \\
\hline$\overline{\text { Gypsum }}$ & 2.787 & $\mathrm{CaSO}_{4} \cdot 2 \mathrm{H}_{2} \mathrm{O}$ & 1.499 & 1.132 \\
\hline Tna & 1.867 & $\mathrm{C}_{6} \mathrm{H}_{3} \mathrm{~N}_{5} \mathrm{O}_{8}$ & 1.499 & 1.158 \\
\hline$\overline{\mathrm{Btx}}$ & 1.87 & $\mathrm{C}_{12} \mathrm{H}_{4} \mathrm{~N}_{8} \mathrm{O}_{10}$ & 1.507 & 1.161 \\
\hline CL20 & 2.044 & $\mathrm{C}_{6} \mathrm{H}_{6} \mathrm{~N}_{12} \mathrm{O}_{12}$ & 1.529 & 1.171 \\
\hline$\overline{\text { Fox-7 }}$ & 1.885 & $\mathrm{C}_{2} \mathrm{H}_{4} \mathrm{~N}_{4} \mathrm{O}_{4}$ & 1.529 & 1.177 \\
\hline$\overline{\text { Graphite }}$ & 3.51 & $\mathrm{C}$ & 1.534 & 1.131 \\
\hline Pyx & 1.75 & $\mathrm{C}_{17} \mathrm{H}_{7} \mathrm{~N}_{11} \mathrm{O}_{16}$ & 1.535 & 1.187 \\
\hline$\overline{\text { Tacot }}$ & 1.85 & $\mathrm{C}_{12} \mathrm{H}_{4} \mathrm{~N}_{8} \mathrm{O}_{8}$ & 1.536 & 1.183 \\
\hline Medips & 1.89 & $\mathrm{C}_{13} \mathrm{H}_{6} \mathrm{~N}_{6} \mathrm{O}_{12} \mathrm{~S}$ & 1.543 & 1.188 \\
\hline Picric Acid & 1.763 & $\mathrm{C}_{6} \mathrm{H}_{3} \mathrm{~N}_{3} \mathrm{O}_{7}$ & 1.559 & 1.205 \\
\hline Dipm & 1.76 & $\mathrm{C}_{12} \mathrm{H}_{6} \mathrm{~N}_{8} \mathrm{O}_{12}$ & 1.569 & 1.212 \\
\hline Dpa & 1.87 & $\mathrm{C}_{12} \mathrm{H}_{6} \mathrm{~N}_{7} \mathrm{O}_{12}$ & 1.579 & 1.215 \\
\hline Tenn & 1.84 & $\mathrm{C}_{10} \mathrm{H}_{4} \mathrm{~N}_{4} \mathrm{O}_{8}$ & 1.587 & 1.223 \\
\hline $\mathrm{Tnr}$ & 1.71 & $\mathrm{C}_{6} \mathrm{H}_{3} \mathrm{~N}_{3} \mathrm{O}_{6}$ & 1.591 & 1.232 \\
\hline Tenpo & 1.86 & $\mathrm{C}_{12} \mathrm{H}_{5} \mathrm{~N}_{5} \mathrm{O}_{9}$ & 1.606 & 1.237 \\
\hline Dingu & 1.908 & $\mathrm{C}_{4} \mathrm{H}_{4} \mathrm{~N}_{6} \mathrm{O}_{6}$ & 1.615 & 1.241 \\
\hline Dimedips & 1.81 & $\mathrm{C}_{14} \mathrm{H}_{8} \mathrm{~N}_{6} \mathrm{O}_{12} \mathrm{~S}$ & 1.623 & 1.253 \\
\hline$\overline{\text { Etn }}$ & 1.6 & $\mathrm{C}_{4} \mathrm{H}_{6} \mathrm{~N}_{4} \mathrm{O}_{12}$ & 1.630 & 1.267 \\
\hline Tetryl & 1.73 & $\mathrm{C}_{7} \mathrm{H}_{5} \mathrm{~N}_{5} \mathrm{O}_{8}$ & 1.643 & 1.268 \\
\hline$\overline{\text { Torpex }}$ & 1.81 & $\mathrm{C}_{1.8} \mathrm{H}_{2.015} \mathrm{~N}_{1.663} \mathrm{O}_{2.19} 1 \mathrm{Al}_{0.6674}$ & 1.651 & 1.274 \\
\hline Fefo & 1.59 & $\mathrm{C}_{5} \mathrm{H}_{6} \mathrm{~N}_{4} \mathrm{O}_{10} \mathrm{~F}_{2}$ & 1.653 & 1.286 \\
\hline$\overline{\text { Pato }}$ & 1.94 & $\mathrm{C}_{8} \mathrm{H}_{5} \mathrm{~N}_{7} \mathrm{O}_{6}$ & 1.654 & 1.271 \\
\hline Tnpon & 1.68 & $\mathrm{C}_{8} \mathrm{H}_{6} \mathrm{~N}_{4} \mathrm{O}_{10}$ & 1.668 & 1.292 \\
\hline Nitroglycerin (NG) & 1.6 & $\mathrm{C}_{3} \mathrm{H}_{5} \mathrm{~N}_{3} \mathrm{O}_{9}$ & 1.672 & 1.300 \\
\hline Pam & 1.78 & $\mathrm{C}_{6} \mathrm{H}_{4} \mathrm{~N}_{3} \mathrm{O}_{6}$ & 1.679 & 1.297 \\
\hline$\overline{\text { Datb }}$ & 1.8 & $\mathrm{C}_{6} \mathrm{H}_{5} \mathrm{~N}_{5} \mathrm{O}_{6}$ & 1.709 & 1.318 \\
\hline$\overline{\mathrm{Dadn}}$ & 1.732 & $\mathrm{C}_{4} \mathrm{H}_{4} \mathrm{~N}_{4} \mathrm{O}_{5}$ & 1.719 & 1.320 \\
\hline$\overline{\text { Tnn }}$ & 1.71 & $\mathrm{C}_{10} \mathrm{H}_{5} \mathrm{~N}_{3} \mathrm{O}_{6}$ & 1.729 & 1.339 \\
\hline$\overline{\mathrm{Cs}}$ & 1.04 & $\mathrm{C}_{10} \mathrm{H}_{5} \mathrm{ClN}_{2}$ & 1.737 & 1.388 \\
\hline Petn & 1.77 & $\mathrm{C}_{5} \mathrm{H}_{8} \mathrm{~N}_{4} \mathrm{O}_{12}$ & 1.744 & 1.347 \\
\hline$\overline{\text { Pentolite }}$ & 1.68 & $\mathrm{C}_{2.33} \mathrm{H}_{2.37} \mathrm{~N}_{1.29} \mathrm{O}_{3.22}$ & 1.753 & 1.357 \\
\hline$\overline{\text { Egdn }}$ & 1.49 & $\mathrm{C}_{2} \mathrm{H}_{4} \mathrm{~N}_{2} \mathrm{O}_{6}$ & 1.756 & 1.371 \\
\hline TNT & 1.654 & $\mathrm{C}_{7} \mathrm{H}_{5} \mathrm{~N}_{3} \mathrm{O}_{6}$ & 1.756 & 1.362 \\
\hline Tatb & 1.93 & $\mathrm{C}_{6} \mathrm{H}_{6} \mathrm{~N}_{6} \mathrm{O}_{6}$ & 1.758 & 1.349 \\
\hline$\overline{\text { Semtex H }}$ & 1.5 & $\mathrm{C}_{4} \mathrm{H}_{7} \mathrm{~N}_{5} \mathrm{O}_{9}$ & 1.766 & 1.377 \\
\hline$\overline{\text { Picratol }}$ & 1.63 & $\mathrm{C}_{2.748} \mathrm{H}_{2.325} \mathrm{~N}_{1.48} \mathrm{O}_{2.749}$ & 1.766 & 1.371 \\
\hline$\overline{\text { Cyclotol-50/50 }}$ & 1.63 & $\mathrm{C}_{2.22} \mathrm{H}_{2.45} \mathrm{~N}_{2.01} \mathrm{O}_{2.67}$ & 1.773 & 1.376 \\
\hline Octol-60/40 & 1.8 & $\mathrm{C}_{2.04} \mathrm{H}_{2.5} \mathrm{~N}_{2.15} \mathrm{O}_{2.68}$ & 1.775 & 1.369 \\
\hline HMX & 1.91 & $\mathrm{C}_{4} \mathrm{H}_{8} \mathrm{~N}_{8} \mathrm{O}_{8}$ & 1.787 & 1.373 \\
\hline Arsanilic Acid & 1.957 & $\mathrm{C}_{6} \mathrm{H}_{8} \mathrm{AsNO}_{3}$ & 1.788 & 1.390 \\
\hline Keto-RDX & 1,867 & $\mathrm{C}_{3} \mathrm{H}_{6} \mathrm{~N}_{6} \mathrm{O}_{6}$ & 1.788 & 1.375 \\
\hline$\overline{\mathrm{Rdx}}$ & 1.82 & $\left(\mathrm{CH}_{2}-\mathrm{N}-\mathrm{NO}_{2}\right)_{3}$ & 1.788 & 1.378 \\
\hline $\mathrm{C} 4$ & 1.68 & $\mathrm{C}_{3} \mathrm{H}_{6} \mathrm{~N}_{6} \mathrm{O}_{6}$ & 1.789 & 1.386 \\
\hline$\overline{D m}$ & 1.648 & $\mathrm{C}_{12} \mathrm{H}_{9} \mathrm{AsClN}$ & 1.789 & 1.405 \\
\hline $\mathrm{Lx}-04$ & 1.86 & $\mathrm{C}_{1.55} \mathrm{H}_{2.58} \mathrm{~N}_{2.3} \mathrm{O}_{2.3} \mathrm{~F}_{0.52}$ & 1.796 & 1.381 \\
\hline Com B & 1.72 & $\mathrm{C}_{2.03} \mathrm{H}_{2.64} \mathrm{~N}_{2.18} \mathrm{O}_{2.67}$ & 1.801 & 1.392 \\
\hline Tfna & 1,692 & $\mathrm{C}_{5} \mathrm{H}_{7} \mathrm{~N}_{4} \mathrm{O}_{6} \mathrm{~F}_{3}$ & 1.819 & 1.409 \\
\hline $\mathrm{Hbx}-1$ & 1.72 & $\mathrm{C}_{2.068} \mathrm{H}_{2.83} \mathrm{~N}_{1.586} \mathrm{O}_{2.085} \mathrm{Al}_{0.63}$ & 1.820 & 1.408 \\
\hline$\overline{\text { Df }}$ & 1.3595 & $\mathrm{CH}_{3} \mathrm{~F}_{2} \mathrm{PO}$ & 1.846 & 1.456 \\
\hline Fivonite & 1.59 & $\mathrm{C}_{9} \mathrm{H}_{12} \mathrm{~N}_{4} \mathrm{O}_{13}$ & 1.868 & 1.451 \\
\hline$\overline{\mathrm{Pbx}-9011}$ & 1.77 & $\mathrm{C}_{1.73} \mathrm{H}_{3.18} \mathrm{~N}_{2.45} \mathrm{O}_{2.6} 1$ & 1.892 & 1.460 \\
\hline Nylon & 1.15 & $\mathrm{C}_{12} \mathrm{H}_{6} \mathrm{~N}_{2} \mathrm{O}_{4}$ & 1.897 & 1.502 \\
\hline Tnx & 1.69 & $\mathrm{C}_{8} \mathrm{H}_{7} \mathrm{~N}_{3} \mathrm{O}_{6}$ & 1.900 & 1.471 \\
\hline Petrin & 1.54 & $\mathrm{C}_{5} \mathrm{H}_{9} \mathrm{~N}_{3} \mathrm{O}_{10}$ & 1.907 & 1.485 \\
\hline Tpeon & 1.56 & $\mathrm{C}_{15} \mathrm{H}_{24} \mathrm{~N}_{8} \mathrm{O}_{26}$ & 1.909 & 1.484 \\
\hline Dina & 1.488 & $\mathrm{C}_{4} \mathrm{H}_{8} \mathrm{~N}_{4} \mathrm{O}_{8}$ & 1.911 & 1.490 \\
\hline$\overline{P v n}$ & 1.6 & $\mathrm{C}_{2} \mathrm{H}_{3} \mathrm{NO}_{3}$ & 1.931 & 1.500 \\
\hline $\mathrm{Dc}$ & 1.3338 & $\mathrm{C}_{13} \mathrm{H}_{10} \mathrm{AsN}$ & 1.953 & 1.558 \\
\hline Tnms & 1.6 & $\mathrm{C}_{9} \mathrm{H}_{9} \mathrm{~N}_{3} \mathrm{O}_{7}$ & 1.976 & 1.535 \\
\hline$\overline{\text { Hnto }}$ & 1.82 & $\mathrm{C}_{2} \mathrm{~N}_{6} \mathrm{H}_{6} \mathrm{O}_{3}$ & 1.981 & 1.525 \\
\hline Amatol-60/40 & 1.6 & $\mathrm{C}_{1.23} \mathrm{H}_{3.88} \mathrm{~N}_{2.03} \mathrm{O}_{3.31}$ & 1.993 & 1.547 \\
\hline$\overline{A c}$ & 0.687 & $\mathrm{HCN}$ & 2.009 & 1.714 \\
\hline Pps & 1.43 & $\mathrm{C}_{6} \mathrm{H}_{4} \mathrm{~S}$ & 2.026 & 1.609 \\
\hline Edna & 1.65 & $\mathrm{C}_{2} \mathrm{H}_{6} \mathrm{~N}_{4} \mathrm{O}_{4}$ & 2.028 & 1.571 \\
\hline$\overline{\mathrm{Adnt}}$ & 1.497 & $\mathrm{C}_{7} \mathrm{H}_{7} \mathrm{~N}_{3} \mathrm{O}_{4}$ & 2.034 & 1.584 \\
\hline$\overline{\text { Degn }}$ & 1.38 & $\mathrm{C}_{4} \mathrm{H}_{8} \mathrm{~N}_{2} \mathrm{O}_{7}$ & 2.060 & 1.613 \\
\hline Daf & 1.77 & $\mathrm{C}_{2} \mathrm{~N}_{4} \mathrm{H}_{4} \mathrm{O}$ & 2.073 & 1.597 \\
\hline$\overline{\text { Hd }}$ & 1.333 & $\mathrm{C}_{4} \mathrm{H}_{8} \mathrm{C}_{12} \mathrm{~S}$ & 2.088 & 1.603 \\
\hline Pvc & 1.38 & $\mathrm{C}_{2} \mathrm{H}_{3} \mathrm{Cl}$ & 2.105 & 1.533 \\
\hline Polyester & 1.4 & $\mathrm{C}_{3} \mathrm{H}_{2} \mathrm{O}$ & 2.138 & 1.676 \\
\hline Dacron & 1.37 & $\mathrm{C}_{10} \mathrm{H}_{8} \mathrm{O}_{4}$ & 2.208 & 1.730 \\
\hline $\mathrm{Cn}$ & 1.3 & $\mathrm{C}_{8} \mathrm{H}_{7} \mathrm{ClO}$ & 2.223 & 1.751 \\
\hline Aspirin & 1.4 & $\mathrm{C}_{9} \mathrm{H}_{8} \mathrm{O}_{4}$ & 2.247 & 1.756 \\
\hline Melamine & 0.72 & $\mathrm{C}_{3} \mathrm{H}_{6} \mathrm{~N}_{6}$ & 2.249 & 1.828 \\
\hline Kevlar & 1.44 & $\mathrm{C}_{14} \mathrm{~N}_{2} \mathrm{O}_{2} \mathrm{H}_{10}$ & 2.250 & 1.755 \\
\hline Pppt & 1.45 & $\mathrm{C}_{14} \mathrm{H}_{10} \mathrm{~N}_{2} \mathrm{O}_{2}$ & 2.250 & 1.755 \\
\hline
\end{tabular}




\begin{tabular}{|c|c|c|c|c|}
\hline Material & Density & Chemical Formula & $\mathbf{R}_{1}$ & $\mathbf{R}_{2}$ \\
\hline Tagn & 1.5 & $\mathrm{CH}_{9} \mathrm{~N}_{7} \mathrm{O}_{3}$ & 2.266 & 1.763 \\
\hline Anfo-6/94 & 0.88 & $\mathrm{C}_{0.43} \mathrm{H}_{5.54} \mathrm{~N}_{2.35} \mathrm{O}_{3.53}$ & 2.286 & 1.837 \\
\hline $\mathrm{Pbt}$ & 1.37 & $\mathrm{C}_{12} \mathrm{H}_{12} \mathrm{O}_{4}$ & 2.444 & 1.913 \\
\hline Pan & 1.184 & $\mathrm{C}_{3} \mathrm{H}_{3} \mathrm{~N}$ & 2.515 & 1.983 \\
\hline Orlon & 1.16 & $\left(\mathrm{CH}_{2}-\mathrm{CHCN}\right)_{\mathrm{n}}$ & 2.517 & 1.985 \\
\hline Hn-1 & 1.086 & $\mathrm{C}_{6} \mathrm{H}_{13} \mathrm{Cl}_{2} \mathrm{~N}$ & 2.526 & 2.014 \\
\hline Mandrax & 1.16 & $\mathrm{C}_{16} \mathrm{H}_{14} \mathrm{~N}_{2} \mathrm{O}$ & 2.526 & 1.988 \\
\hline Aspartame & 1.35 & $\mathrm{C}_{14} \mathrm{H}_{18} \mathrm{~N}_{2} \mathrm{O}_{5}$ & 2.536 & 1.985 \\
\hline Sugar & 0.88 & $\mathrm{C}_{12} \mathrm{H}_{22} \mathrm{O}_{11}$ & 2.558 & 2.052 \\
\hline $\mathbf{H t}$ & 1.263 & $\mathrm{C}_{2.74} \mathrm{H}_{7.923} \mathrm{CL}_{1.065} \mathrm{O}_{0.153} \mathrm{~S}_{0.685}$ & 2.580 & 2.039 \\
\hline Heroin & 1.34 & $\mathrm{C}_{21} \mathrm{H}_{23} \mathrm{NO}_{5}$ & 2.589 & 2.029 \\
\hline Men-II & 1.02 & $\mathrm{C}_{2.06} \mathrm{H}_{7.06} \mathrm{~N}_{1.33} \mathrm{O}_{3.1}$ & 2.608 & 2.072 \\
\hline Wood & 0.593 & $\mathrm{C}_{22} \mathrm{H}_{31} \mathrm{O}_{12}$ & 2.621 & 2.155 \\
\hline Flour & 0.593 & $\mathrm{C}_{6} \mathrm{H}_{12} \mathrm{O}_{6}$ & 2.625 & 2.160 \\
\hline Benzene & 0.8786 & $\mathrm{C}_{6} \mathrm{H}_{6}$ & 2.633 & 2.359 \\
\hline $\mathrm{Bz}$ & 1.33 & $\mathrm{C}_{21} \mathrm{H}_{23} \mathrm{NO}_{3}$ & 2.637 & 2.066 \\
\hline Wool & 1.28 & $\mathrm{C}_{4.25} \mathrm{H}_{7} \mathrm{NO}_{1.75} \mathrm{~S}_{0.125}$ & 2.652 & 2.081 \\
\hline Pct & 1.23 & $\mathrm{C}_{16} \mathrm{H}_{18} \mathrm{O}_{4}$ & 2.658 & 2.090 \\
\hline Morphine & 1.31 & $\mathrm{C}_{17} \mathrm{H}_{19} \mathrm{NO}_{3}$ & 2.676 & 2.100 \\
\hline Cocaine & 1.14 & $\mathrm{C}_{17} \mathrm{H}_{21} \mathrm{NO}_{4}$ & 2.716 & 2.145 \\
\hline Rice & 0.702 & $\mathrm{C}_{4.15} \mathrm{H}_{9.42} \mathrm{~N}_{0.1} \mathrm{O}_{4.53}$ & 2.732 & 2.241 \\
\hline Coffee & 0.4 & $\mathrm{C}_{2.24} \mathrm{H}_{3.61} \mathrm{~N}_{0.1} \mathrm{O}_{1.24}$ & 2.742 & 2.323 \\
\hline Ghb & 1.21 & $\mathrm{C}_{4} \mathrm{H}_{8} \mathrm{O}_{3}$ & 2.759 & 2.169 \\
\hline$M D A$ & 1.08 & $\mathrm{C}_{10} \mathrm{H}_{13} \mathrm{NO}_{2}$ & 2.777 & 2.198 \\
\hline Tatp & 1.18 & $\mathrm{C}_{9} \mathrm{H}_{18} \mathrm{O}_{6}$ & 2.839 & 2.236 \\
\hline$L S D$ & 1.21 & $\mathrm{C}_{20} \mathrm{H}_{25} \mathrm{~N}_{3} \mathrm{O}$ & 2.875 & 2.263 \\
\hline Acetamide & 1.16 & $\mathrm{CH}_{3} \mathrm{CONH}_{2}$ & 2.890 & 2.277 \\
\hline $\mathbf{V x}$ & 1.06 & $\mathrm{C}_{7} \mathrm{H}_{18} \mathrm{NO}_{2} \mathrm{PS}$ & 2.956 & 2.377 \\
\hline ABS Plastic & 1.04 & $\left(\mathrm{C}_{8} \mathrm{H}_{8} \cdot \mathrm{C}_{4} \mathrm{H}_{6} \cdot \mathrm{C}_{3} \mathrm{H}_{3} \mathrm{~N}\right)_{\mathrm{n}}$ & 2.974 & 2.355 \\
\hline Fentanyl & 1.035 & $\mathrm{C}_{22} \mathrm{H}_{28} \mathrm{~N}_{2} \mathrm{O}$ & 2.995 & 2.374 \\
\hline Nicotine & 1.01 & $\mathrm{C}_{10} \mathrm{H}_{14} \mathrm{~N}_{2}$ & 3.040 & 2.412 \\
\hline Methanol & 0.7918 & $\mathrm{CH}_{3} \mathrm{OH}$ & 3.058 & 2.463 \\
\hline Methadone & 0.93 & $\mathrm{C}_{2} 1 \mathrm{H}_{27} \mathrm{NO}$ & 3.077 & 2.455 \\
\hline Ppma & 1.08 & $\mathrm{C}_{7} \mathrm{H}_{12} \mathrm{O}_{2}$ & 3.108 & 2.456 \\
\hline Det & 1.2 & $\mathrm{C}_{14} \mathrm{H}_{20} \mathrm{~N}_{2}$ & 3.129 & 2.456 \\
\hline Water & 1 & $\mathrm{H}_{2} \mathrm{O}$ & 3.143 & 2.494 \\
\hline Dxm & 0.95 & $\mathrm{C}_{18} \mathrm{H}_{25} \mathrm{NO}$ & 3.147 & 2.505 \\
\hline Amphetamine & 0.913 & $\mathrm{C}_{9} \mathrm{H}_{13} \mathrm{~N}$ & 3.220 & 2.569 \\
\hline Dipt & 1.05 & $\mathrm{C}_{16} \mathrm{H}_{24} \mathrm{~N}_{2}$ & 3.235 & 2.557 \\
\hline $\mathrm{Pbma}$ & 1.053 & $\mathrm{C}_{8} \mathrm{H}_{14} \mathrm{O}_{2}$ & 3.258 & 2.577 \\
\hline Phma & 1.007 & $\mathrm{C}_{10} \mathrm{H}_{18} \mathrm{O}_{2}$ & 3.319 & 2.630 \\
\hline$P C P$ & 0.89 & $\mathrm{C}_{17} \mathrm{H}_{25} \mathrm{~N}$ & 3.334 & 2.663 \\
\hline Ethanol & 0.789 & $\mathrm{C}_{2} \mathrm{H}_{6} \mathrm{O}$ & 3.657 & 2.938 \\
\hline Beeswax & 0.95 & $\mathrm{C}_{15} \mathrm{H}_{31} \mathrm{CO}_{2} \mathrm{C}_{30} \mathrm{H}_{61}$ & 3.909 & 3.102 \\
\hline Polyethylene & 0.94 & $(\mathrm{CH} 2)_{\mathrm{n}}$ & 3.919 & 3.108 \\
\hline
\end{tabular}

Based on the uncertainty values $\left(1 \%\right.$ on $I_{n} / I_{n}^{0}$ value and 0.1 on $I_{g} / I_{g}^{0}$ value), Table 2 shows the range for $R$ value in each case for the materials which were illustrated in Fig. 3. In order to evaluate the effectiveness of the FNGR systems comparison with typical X-rays systems, the numbers of materials with overlapped $\mathrm{R}$ values have been calculated. Table 3 shows the percentage of materials with overlapped $R$ values both for all materials and between of illicit and not illicit materials. The ${ }^{252} \mathrm{Cf}$ neutrons $/{ }^{137} \mathrm{Cs}$ combination offers better discrimination with values $2.33 \%$ and $1.18 \%$ while the ${ }^{252} \mathrm{Cf}$ neutrons $/{ }^{252} \mathrm{Cf}$ gammas combination gives similar results $(3.02 \%$ and $1.96 \%$ respectively). For comparison the typical dual system based on a 4 and $9 \mathrm{MeV}$ Bremsstrahlung spectra X-ray sources has $9.75 \%$ and $6.01 \%$. The combination of the two FNGR systems improves more the effectiveness of the simulated system $(1.77 \%$ and $0.89 \%$ correspondingly).

Table 2. The range for $\mathrm{R}$ value for 24 materials.

\begin{tabular}{l|l|l|l|l|l|l}
\hline Material & $\mathbf{R}_{\mathbf{1}}$ & $\mathbf{R}_{\mathbf{1}} \mathbf{m}$ - $\mathbf{m a x}$ & $\mathbf{R}_{\mathbf{2}}$ & $\mathbf{R}_{\mathbf{2}} \mathbf{m} \mathbf{n}-\mathbf{m a x}$ & $\mathbf{R}_{\mathbf{3}}$ & $\mathbf{R}_{\mathbf{3}} \mathbf{m} \mathbf{m}-\mathbf{m a x}$ \\
\hline Uranium & 0.222 & $0.221-0.223$ & 0.093 & $0.093-0.094$ & 1.0835 & $1.0803-1.0807$ \\
Plutonium & 0.330 & $0.329-0.331$ & 0.134 & $0.133-0.134$ & 1.0805 & $1.0833-1.0838$ \\
Lead & 0.347 & $0.346-0.349$ & 0.178 & $0.178-0.179$ & 1.1109 & $1.0841-1.0846$ \\
Gold & 0.392 & $0.391-0.393$ & 0.193 & $0.192-0.193$ & 1.0843 & $1.1105-1.1113$ \\
Copper & 0.645 & $0.643-0.648$ & 0.448 & $0.447-0.450$ & 1.2359 & $1.3650-1.3542$ \\
Iron & 0.683 & $0.680-0.685$ & 0.480 & $0.478-0.481$ & 1.2560 & $1.2352-1.2365$ \\
Aluminum & 1.023 & $1.016-1.030$ & 0.776 & $0.770-0.781$ & 1.3255 & $1.2521-1.2536$ \\
Gypsum & 1.499 & $1.492-1.506$ & 1.132 & $1.127-1.138$ & 1.2975 & $1.2955-1.2994$ \\
Tetryl & 1.652 & $1.632-1.654$ & 1.268 & $1.259-1.276$ & 1.3585 & $1.3233-1.3278$ \\
Nitroglycerin & 1.672 & $1.660-1.684$ & 1.300 & $1.291-1.309$ & 1.3555 & $1.3518-1.3592$ \\
Petn & 1.744 & $1.733-1.756$ & 1.347 & $1.339-1.355$ & 1.3571 & $1.3538-1.3604$ \\
\hline \hline
\end{tabular}




\begin{tabular}{|c|c|c|c|c|c|c|}
\hline TNT & 1.756 & $1.744-1.768$ & 1.362 & $1.353-1.371$ & 1.3583 & $1.3492-1.3659$ \\
\hline$\underline{\mathrm{HMX}}$ & 1.787 & $1.777-1.798$ & 1.373 & $1.366-1.381$ & 1.3579 & $1.3548-1.3610$ \\
\hline$\underline{\mathrm{Rdx}}$ & 1.788 & $1.777-1.799$ & 1.378 & $1.370-1.386$ & 1.3580 & $1.3548-1.3612$ \\
\hline Nylon & 1.900 & $1.880-1.914$ & 1.502 & $1.489-1.515$ & 1.3599 & $1.3547-1.3619$ \\
\hline Ac & 2.009 & $1.982-2.036$ & 1.714 & $1.692-1.737$ & 1.3575 & $1.3551-1.3620$ \\
\hline Polyester & 2.138 & $2.124-2.152$ & 1.676 & $1.665-1.687$ & 1.3613 & $1.3548-1.3650$ \\
\hline Heroin & 2.589 & $2.574-2.604$ & 2.029 & $2.018-2.041$ & 1.3637 & $1.3561-1.3654$ \\
\hline Cocaine & 2.716 & $2.699-2.734$ & 2.145 & $2.132-2.158$ & 1.3637 & $1.3572-1.3655$ \\
\hline Ghb & 2.759 & $2.742-2.775$ & 2.169 & $2.156-2.181$ & 1.3607 & $1.3587-1.3686$ \\
\hline$L S D$ & 2.875 & $2.858-2.892$ & 2.263 & $2.250-2.275$ & 1.3648 & $1.3595-1.3680$ \\
\hline Methadone & 3.077 & $3.056-3.099$ & 2.455 & $2.438-2.471$ & 1.3657 & $1.3601-1.3694$ \\
\hline Water & 3.143 & $3.123-3.163$ & 2.494 & $2.479-2.509$ & 1.359 & $1.3597-1.3717$ \\
\hline Polyethylene & 3.919 & $3.897-3.941$ & 3.108 & $3.092-3.125$ & 1.3693 & $1.3637-1.3750$ \\
\hline
\end{tabular}

Table 3. The percentage and the number of the pairs with overlapped $\mathrm{R}$ values

\begin{tabular}{|c|c|c|c|c|}
\hline & \multicolumn{3}{|c|}{ Dual Beam } & \multirow{2}{*}{$\begin{array}{l}\begin{array}{l}\text { Triple } \\
\text { beam }\end{array} \\
\mathrm{R}_{1}-\mathrm{R}_{2} \\
1.776 \%\end{array}$} \\
\hline $\begin{array}{l}\text { Case } \\
\text { Overlapped pair } \\
\text { (total) }\end{array}$ & $\begin{array}{l}\mathrm{R}_{1} \\
3.026 \%\end{array}$ & $\begin{array}{l}\mathrm{R}_{2} \\
2.335 \%\end{array}$ & $\begin{array}{l}\mathrm{R}_{3} \\
9.756 \%\end{array}$ & \\
\hline $\begin{array}{l}\text { Overlapped pairs } \\
\text { (between illicit - } \\
\text { not illicit materials) }\end{array}$ & $1.963 \%$ & $1.181 \%$ & $6.018 \%$ & $0.891 \%$ \\
\hline
\end{tabular}

In addition, using two FNGR systems, the simulation facility offers considerable advantage in material discrimination. There are many materials with almost the same R1 or R2 values and the presence of two $\mathrm{R}$ values instead of one affords valuable supplementary information. For example Fig 4 shows some pairs of materials with nearly equal R1 values but different R2 values. The R1 values for these pairs varies from $0.06 \%$ to $0.34 \%$ while the respectively R2 values ranging between $1.72-15.72 \%$. Correspondingly Fig. 5 illustrates materials couples with unequal R1 values and practically same R2 values.

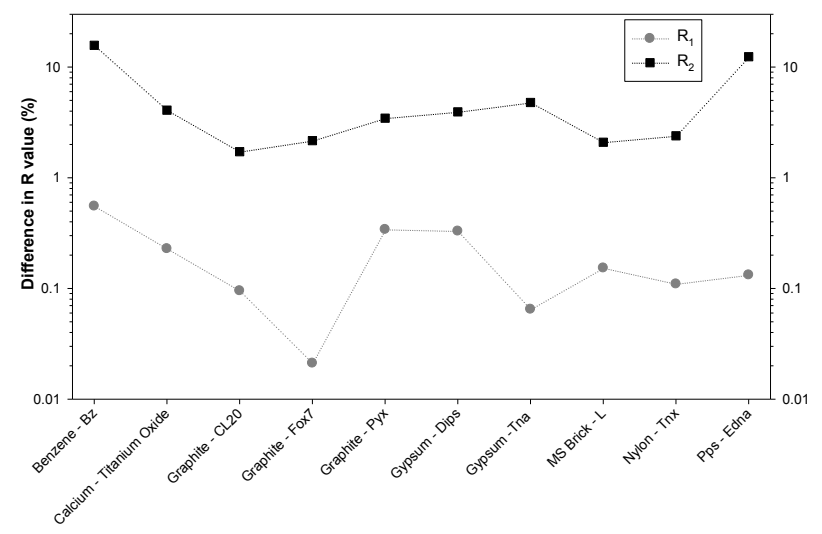

Materials

Fig. 4. Materials with nearly same R1 values but different R2 values.

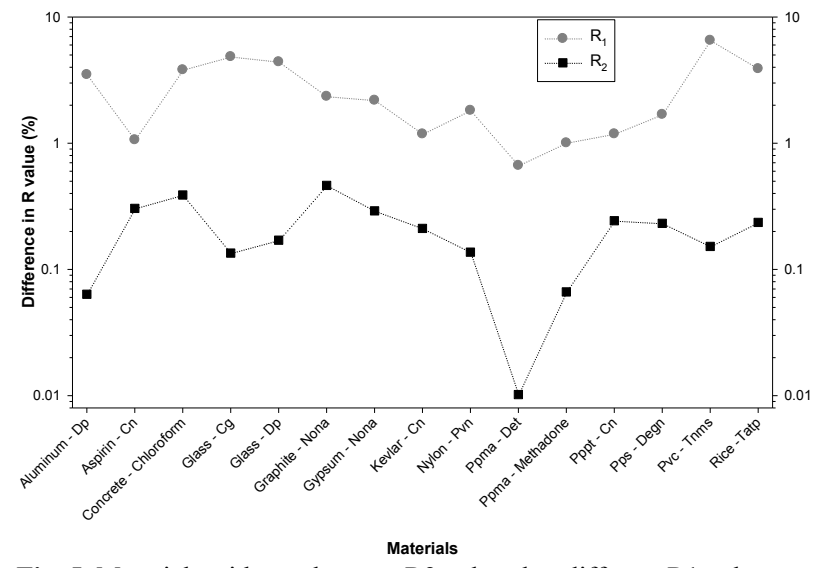

Fig. 5. Materials with nearly same R2 values but different R1 values.

\section{Conclusions}

A simple FNGR system based on a ${ }^{252} \mathrm{Cf}$ and ${ }^{137} \mathrm{Cs}$ isotopic sources evaluated using the MCNP4B Monte Carlo code. The evaluation has been brought into effect on the basis of the $R$ values and the percentage of materials with similar $R$ values was used as gauges of the effectiveness of each source mode. The two dual beam FNGR systems offer very good discriminator capabilities among a wide range of organic threat materials, narcotics and explosives while the use of three sources, instead of two, develops the capability of neutron/gamma combination to separate similar in composition materials.

\section{References}

1. S. Ogorodnikov, V. Petrunin, Phys. Rev .Spec. Top.-Accel. Beams 5 (2002) 104701.

2. Z. Chen, X. Wang, Port Technol. Int. 30 (2006) 163.

3. A. Buffler, Rad. Phys. and Chem. 71 (2004) 853.

4. J.E. Eberhardt, S. Rainey, R.J. Stevens, B.D. Sowerby, J.R. Tickner, Appl. Radiat. Isot. 63 (2005) 179

5.J.E. Eberhardt, Y. Liu, S. Rainey, G.J. Roach, R.J. Stevens, B.D. Sowerby, J.R. Tickner, Fast neutron and gamma-ray interrogation of air cargo containers. International Workshop on Fast Neutron Detectors, Cape Town, April 3-6 2006.

6. WANG X-W, LI J-M, KANG K-J, TANG C-X, ZHANG L, CHEN Z-Q, LI Y-J, ZHONG H-Q, Material Discrimination by High-
Energy X-Ray Dual-Energy Imaging, HIGH ENERGY PHYSICS AND NUCLEAR PHYSICS, Vol. 31, No. 11 Nov., 2007.

7. Y. Liu, B.D. Sowerby, J.R. Tickner, Appl. Radiat. and Isot. 66 (2008) 463.

8. B.D. Sowerby, N.G. Cutmore, Y. Liu, H. Peng, J.R. Tickner, Y. Xie, C. Zong, Recent Developments in Fast Neutron Radiography for the Interrogation of Air Cargo Containers, IAEA Conference, Vienna, 4-8 May 2009.

9. R.C. Runkle, T.A. White, E.A. Miller, J.A. Caggiano, B.A. Collins, Nucl. Instrum. and Meth. A 603 (2009) 510.

10. A. Buffler, J. Tickner, Radiation Measurements 45 (2010) 1186. 
11. J. G. Fantidis, G. E. Nicolaou, Journal of Radioanalytical and Nuclear Chemistry 2952 (2013), 973-977.

12. Briesmeister JF (1997). MCNP4B MCNPTM-A General Monte Carlo N-particle transport code, version 4B LA-12625-M Manual.

13. J. G. Fantidis, G. E. Nicolaou, Nucl. Instrum. and Meth. A 648.1 (2011) 275-284

14. V.V. Verbinski, H. Weber, R.E. Sund (1973) Phys Rev C $7(3): 1173$.

15. Headquarters, Department of the Army Technical ManuaL, Military Explosives, U.S. Government Printing Office: 1995.

16. A. Bailey S. G. Murray, Brassey's World Military Technology Explosives, Propellants and Pyrotechnics, Royal military college of science Shrivenham, UK, 2000.

17. M. Zakikhani, M.S. Dortch, J.A. Gerald, Compilation of Physica and Chemical Properties and Toxicity Benchmarks for Military Range Compounds, US Army Corps of Engineers 2002.

18. J.C. Pennington, K.A. Thorn, L.G. Cox, D.K. MacMillan, S. Yost, R.D. Laubscher, Photochemical Degradation of Composition B and Its Components, US Army Corps of Engineers 2007.

19. http://cameochemicals.noaa.gov

20. http://www.chemindustry.com

21. http://www.powerlabs.org/

22. A. Aziz, The Mujahideen Explosives Handbook, O. P. M. 1998.

23. H. Muthurajan, R. Sivabalan, M.B. Talawar, S.N. Asthana, J. Hazard. Mat A112 (2004).

24. G. Wang, H. Xiao, X. Ju, X. Gong, Propellants, Explos. Pyrotechnics 31(5) (2006) 361.

25. M.Chovancova, S. Zeman, Thermochim.Acta 460 (2007) 67.

26. M.H. Keshavarz, J. Hazard. Mater. 143 (2007) 437.

27. M.H. Keshavarz, J. Hazard. Mater. 147 (2007) 826.

28. M.H. Keshavarz, J. Hazard. Mater. 150 (2008) 387.

29. M. H. Keshavarz, J. Hazard. Mater. 153 (2008) 201

30. M.H. Keshavarz, H.R. Pouretedal, A. Semnani, Chemistry 17 (6) (2008) 470

31. D.M. Badgujar, M.B. Talawar, S.N. Asthana, P.P. Mahulikar, J. Hazard. Mater. 151 (2008) 289.

32. M.H. Keshavarz, J. Hazard. Mater. 166 (2009) 762.
33. M.H. Keshavarz, J. Hazard. Mater. 166 (2009) 1296.

34. S. Fordham, High Explosives and Propellants, Pergamon Press, Oxford, England, 1980.

35. T. Urbanski, The Chemistry and Technology of Explosives, vol. 14, Pergamon Press, Oxford, England, 1985.

36. J. Akhavan, The Chemistry of Explosives, The Royal Society of Chemistry, 1987.

37. W. Wallace, FMX The revised Black Book A Guide to FieldManufactured Explosives, Paladin Press, 1995.

38. R. Meyer, J. Köhler, A. Homburg, Explosives, Wiley-VCH Verlag $\mathrm{GmbH}$, Weinheim, Germany, 2002.

39. N. Kubota, Propellants and Explosives, Thermochemical Aspects of Cobustion, Wiley-VCH Verlag GmbH, Weinheim, Germany, 2002.

40. International Narcotics Control Board, List Of Narcotic Drugs Under International Control, Vienna International Centre, Vienna 2004 .

41. R.S. Vardanyan and V.J. Hruby, Synthesis of Essential Drugs, Elsevier, 2006

42. K. Steven, Drug Abuse Handbook, CRC press 1998.

43. H.R. Kranzler, P. Korsmeyer, Encyclopedia Of Drugs, Alcohol \& Addictive Behavior, volume 1-4, Macmillan Reference USA, Gale, Cengage Learning 2009.

44. http://www.erowid.org/chemicals/

45. Army, Marine Corps, Navy, Air Force, Potential Military Chemical/Biological Agents and Compounds, Active Army, Army National Guard, and US Army Reserve, January 2005.

46. www.nicnas.gov.au

47. http://www.engineeringtoolbox.com/

48. http://www.azom.com/default.asp

49. http://www.agcc.jp/2005/en/index.html

50. http://www.polymerprocessing.com/index.html

51. S. Joseph, Polymeric Materials Encyclopedia, CRC press 1996.

52. D.K. Platt, Engineering and High Performance Plastics, Rapra Technology, Shawbury, UK., 2003.

53. S.C. Gujrathi, J.M. D'auria, Nucl. Instrum. and Meth. 100 (1972) 445. 